\title{
MULTI-SCALE STATISTICAL DETECTION AND BALLISTIC IMAGING THROUGH TURBID MEDIA
}

\author{
Sina Farsiu \\ Eye Research Center, Duke University \\ Durham, NC 27710, USA. \\ sina.farsiu@duke.edu
}

\author{
Peyman Milanfar \\ EE Department, University of California \\ Santa Cruz, CA 95064, USA. \\ milanfar@ee.ucsc.edu
}

\begin{abstract}
We exploit recent advances in the physical design of fast optical systems which enable active imaging with "ballistic" light. In this modality, fast bursts of optical energy are propagated into a medium, and the ballistic component of light (which travels with minimal diffusive distortion) is detected after transmission through the target and the medium. To improve the detection rate of the common single pixel optimal detectors, we exploit sampling at a diversity of locations in space, and develop a multi-scale algorithm based upon the Generalized Likelihood Ratio Test (GLRT) framework, which takes advantage of the spatial correlation of nearby samples. Experimental results show that objects of different size and shape that are completely unrecognizable using the common single pixel detection techniques, are detectable with very high accuracy using the said multi-scale GLRT technique.
\end{abstract}

Index Terms- Ballistic Photons, Poisson Statistics, Adaptive Reconstruction, GLRT, Coherent Imaging, Turbid Media.

\section{INTRODUCTION}

High resolution imaging and detection of objects hidden in a turbid (scattering) medium have long been challenging and important problems with many industrial, military, and medical applications. While turbid media such as fog, smoke, haze, or body tissue are virtually transparent to radar range electromagnetic waves, the resolution of radar-based imaging systems is often insufficient for many practical applications. On the other hand, while the resolution of imaging systems using ultra short wavelengths (e.g. X-rays) is very desirable, there exist potential health hazards for imaging subjects and technicians alike.

As an alternative, imaging systems working in the optical/ infra-red spectrum range (laser scanners) are potentially able to produce high resolution images without the likely health hazards. Unfortunately, even a very thin and powerful collimated laser beam quickly diffuses as it travels in turbid me-

WE WISH TO THANK PROFS. BENJAMIN FRIEDLANDER, INES DELFINO, HEIKE LISCHKE, AND MOHAMMAD ALRUBAIEE FOR PROVIDING INVALUABLE INFORMATION AND DATA THROUGHOUT THIS PROJECT. THIS WORK WAS SUPPORTED IN PART BY DARPA/AFOSR GRANT NUMBER FA9550-06-1-0047. APPROVED FOR PUBLIC RELEASE, DISTRIBUTION UNLIMITED. dia, similar to a car's headlights in fog. Therefore, a naive approach to optical imaging of objects hidden inside a turbid medium results in very blurry images, where targets are often indistinguishable from each other or the background.

Fortunately, the advent of the new tunable solid state lasers and ultra-fast optical detectors has enabled us to acquire highquality images through turbid media where the resolution is only limited by diffraction. While many efficient imaging systems for capturing high-resolution images through turbid media have been proposed through the years, in this paper we mainly focus on ultra-fast time-gated or coherent imaging systems first introduced in [1].

Ultra-fast time-gated imaging is based on scanning the region of interest (ROI) point-by-point by sending very fast bursts of optical energy (laser pulses) and detecting the unscattered (coherent) photons that have passed through the medium or reflected from the object. Although most of the photons in a laser pulse are either randomly scattered (losing their coherence) or absorbed as they travel through turbid media, across short distances, a few photons keep their coherence and pass through in straight lines without being scattered. These coherent photons are commonly referred to as the ballistic photons. Aside from the diffusive and ballistic photons, the photons that are slightly scattered retaining some degree of coherence are referred to as snake photons. Since the diffusive and ballistic photons have different path lengths, a femto-second laser pulse generator and an ultra fast time gate can be paired to separate the relatively slow (delayed) diffusive photons from the ballistic ones.

In what follows in this paper, we focus on studying and improving the performance of ballistic imaging systems. In Section 2, we describe a statistical model for the signal and noise in a typical ballistic imaging scenario. In Section 3, we study optimal single pixel detection systems and show that better detection rates are achievable using a multi-pixel detection technique which is based on the GLRT principle. Section 4 concludes this paper.

\section{STATISTICAL MODEL FOR BALLISTIC IMAGING SYSTEMS}

To have a better understanding of the practical issues involved in photon limited imaging via ballistic systems, let us con- 
sider the imaging system described in [2], where the pumped Ti:Sapphire laser radiates $800 \mathrm{~nm}$ pulses at a repetition rate of $1 \mathrm{kHz}$ and an average power of $60 \mathrm{~mW}$. It is easy to show that the number of photons in each packet of energy (pulse) is computed as

$I_{0}=\frac{\text { Pulse Energy }}{\text { Photon Energy }}=\frac{\frac{60 \times 10^{-3} \times 1 s}{1000}}{2.4830 \times 10^{-19}}=2.4164 \times 10^{14}$.

Due to the statistical nature of pulse propagation, as a laser beam travels through a diffusive medium, it is possible that some of the photons emerge without being scattered. By selecting these unscattered "ballistic" photons, and rejecting the scattered (diffused) ones, it is possible to obtain nonblurred images which are the sharp shadows of targets buried in the diffusive medium.

As expected, in relatively long distances, the number of detected ballistic photons is extremely small. Indeed, Beer's Law dictates an exponential relationship between the intensity of the transmitted light, and that of the ballistic component as

$$
I_{b}=I_{0} \exp \left(-\frac{d}{L}\right) \text {. }
$$

In this expression, $I_{0}$ is the number of the generated photons in one laser pulse before entering the turbid medium, $I_{b}$ is the number of the ballistic photons which survive traveling through the medium, $d$ is the distance traveled through the medium, $L=\frac{1}{\mu_{t}}$ is the mean free path (MFP) length (average distance photons travel before being scattered), and $\mu_{t}$ is the medium extinction factor. From (1) and (2), it is clear that for typical laser powers, it is fairly unlikely that any ballistic photon survive imaging scenarios where the ratio of $d / L$ is larger than $\sim 30$ MFP.

The exponential drop in the number of received photons is the main prohibitive factor for using such high-resolution optical imaging systems across long distances. In such imaging scenarios, we are forced to rely on the less informative snake and diffusive photons. In [3], an accurate yet computationally manageable mathematical model for diffusive light propagation in turbid media is presented. An example of such imaging modality and experimental analysis is presented in [4] and some excellent literature surveys on the subject of diffusive imaging systems are presented in [5]. However, imaging systems that are able to time-resolve both ballistic and diffusive photons are rather expensive and are not discussed in this paper. Here, we focus on imaging systems that detect ballistic photons only. We exploit these statistical studies to improve the performance of ballistic imaging systems even in long distances where the signal power is weak.

It is important to note that due to the stochastic nature of photon propagation, $I_{b}$, calculated in (2), is merely the expected value of a Poisson random variable that estimates the number of surviving ballistic photons. Moreover, we assume that the received signal at the detector is contaminated with some amount of independent Poisson noise due to shot noise and other degrading effects. Therefore, since the received signal at the detector is the unweighted summation of two Poisson random variables, it can be modeled as a Poisson random process with the following expected value

$$
I=I_{0} e^{-\mu_{t} d}+X_{e}=X_{s}+X_{e},
$$

where $X_{e}$ and $X_{s}$ are the expected values of the noise and signal, respectively.

Considering such imaging model, the probability density function of the received signal is given by

$$
f\left(\underline{y} \mid X_{\underline{s}}+X_{\underline{e}}\right)=\prod_{k=1}^{N} \frac{e^{-\left(X_{e_{k}}+X_{s_{k}}\right)}\left(X_{e_{k}}+X_{s_{k}}\right)^{y_{k}}}{y_{k} !},
$$

where $y_{k}$ is the k-th measurement, $y=\left[y_{1}, y_{2}, \ldots, y_{k}, \ldots, y_{N}\right]^{T}$, $X_{\underline{e}}=\left[X_{e_{1}}, X_{e_{2}}, \ldots, X_{e_{k}}, \ldots, X_{e_{N}}\right]^{T}, \quad$ and $X_{\underline{s}}=\left[X_{s_{1}}, X_{s_{2}}, \ldots, X_{s_{k}}, \ldots, X_{s_{N}}\right]^{T}$. Note that the laser emits thousands of pulses per second and in practical implementation each spatial position is measured $N$ times to improve the quality of estimation, and therefore the model in (3) is presented in the vector form. Since the average power of laser or the detector (and medium) characteristics are assumed not to be changing abruptly, to simplify notations, we assume that $X_{e_{1}}=X_{e_{2}}=\ldots=X_{e_{N}}=X_{e}$, and $X_{s_{1}}=X_{s_{2}}=\ldots=$ $X_{s_{N}}=X_{s}$ (extension to the more general time-varying signal and noise case is straight forward).

\section{OPTIMAL DETECTION OF OPAQUE OBJECTS IN TURBID MEDIA}

In this section, assuming that the medium, laser, target, and turbid medium are accurately calibrated, we present the statistical optimal detectors of opaque objects hidden in a turbid medium.

\subsection{Single Pixel Optimal Detection}

In this subsection, we study the Neyman-Pearson (N.P.) type statistical test [6] for detecting opaque objects hidden in a turbid medium. In this test, we basically compare the likelihood of the following two scenarios:

- $\mathbb{H}_{0}$ : An opaque object is hidden in the scattering medium, blocking the laser pulse ( i.e. measurements contain only noise).

- $\mathbb{H}_{1}$ : No opaque object exists in the propagation line of the laser pulse (i.e. measurements contain noise plus attenuated laser pulse).

The probability density function of these two scenarios when such tests are repeated $N$ times are given by

$$
\begin{aligned}
& \mathbb{H}_{0} \quad: \quad f\left(\underline{y} \mid X_{e}\right)=\prod_{k=1}^{N} \frac{e^{-\left(X_{e}\right)}\left(X_{e}\right)^{y_{k}}}{y_{k} !}, \\
& \mathbb{H}_{1} \quad: \quad f\left(\underline{y} \mid X_{s}+X_{e}\right)=\prod_{k=1}^{N} \frac{e^{-\left(X_{e}+X_{s}\right)}\left(X_{e}+X_{s}\right)^{y_{k}}}{y_{k} !},(4)
\end{aligned}
$$


and therefore the N.P. test is derived by comparing the log likelihood ratio to a threshold as:

$$
\begin{aligned}
& \log \prod_{k=1}^{N}\left(\frac{\frac{e^{-\left(X_{e}+X_{s}\right)}\left(X_{e}+X_{s}\right)^{y_{k}}}{y_{k} !}}{\frac{e^{-X_{e}}\left(X_{e}\right)^{y_{k}}}{y_{k} !}}\right) \underset{\mathbb{H}_{0}}{\stackrel{\mathbb{H}_{1}}{\gtrless}} \gamma \\
& \Longrightarrow \sum_{k=1}^{N} y_{k} \underset{\mathbb{H}_{0}}{\stackrel{\mathbb{H}_{1}}{\gtrless}} \frac{\log (\gamma)+N X_{s}}{\log \left(\frac{X_{e}+X_{s}}{X_{e}}\right)}=\gamma^{\prime} .
\end{aligned}
$$

Noting that $\sum_{k=1}^{N} y_{k}$ is yet another poisson process, the probabilities of false alarm $\left(P_{F A}\right)$ and detection $\left(P_{D}\right)$ are computed as

$$
\begin{gathered}
P_{F A}=P\left\{\sum_{k=1}^{N} y_{k}>\gamma^{\prime} \mid \mathbb{H}_{0}\right\}=1-\sum_{k=0}^{\gamma^{\prime}} \frac{e^{-N X_{e}}\left(N X_{e}\right)^{k}}{k !}, \\
P_{D}=1-\sum_{k=0}^{\gamma^{\prime}} \frac{e^{-N X_{e}-N X_{S}}\left(N X_{e}+N X_{S}\right)^{k}}{k !} .
\end{gathered}
$$

\subsection{Multi-pixel GLRT Detection}

As explained in Section 1, in ballistic imaging the field of view is scanned at multiple points to create a 2-D image of the objects in the ROI. In this section, we propose an effective algorithm that exploits the spatial correlation of the nearby samples in a multi-pixel imaging scenario to improve on the performance of the single pixel optimal detectors developed in the previous section.

The proposed multi-pixel detection technique generalizes the single pixel detection techniques and preforms optimal tests on "super-pixels", which are the collective intensities of a set of neighboring pixels in size and shape of the hidden objects. However, since in general the size and shape of the hidden objects is not known a priori, we develop a GLRT based algorithm that simultaneously tests the existence, and estimates the shape and size of the objects hidden in turbid media.

The outline of the proposed GLRT algorithm is illustrated by an example in Fig.1. First, for a given (fixed) false alarm rate the optimal detectors developed in the previous section are exploited to test the existence or absence of objects at each individual pixel. As an illustrative example, this test is applied to the central pixel (shaded) of Fig.1(a), where the measured pixel value (0.4) is compared to the N.P. test threshold (0.5). Of course, the greater the distance of the measurement from the threshold, the more confident we are in the accuracy of the test result. Next, we integrate the gray-level values of all immediate neighboring pixels, and in effect consider them as one "super-pixel", as illustrated in Fig.1(b). Since the false alarm rate is fixed for all scales, the decision threshold is different than the threshold calculated in the previous step, which is recalculated based on the grayvalue of the superpixel. In the next steps, we repeat this process by fixing the

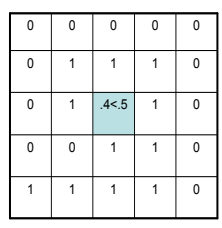

a

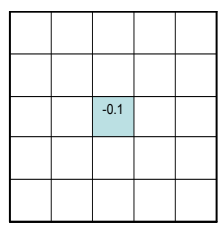

d

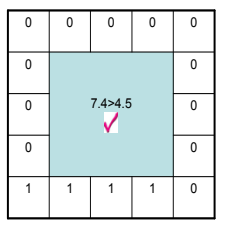

b

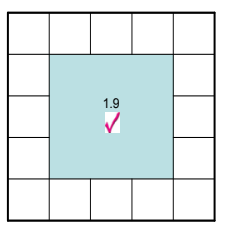

e

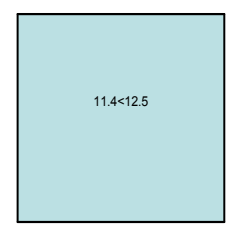

c

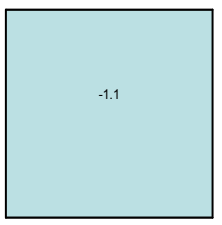

f
Fig. 1. An illustrative example showing the outline of the proposed multi-scale GLRT algorithm. a: Scale 1 measurement. b,c: Scales 2,3 super-pixels, respectively. d-f: Confidence values for scales $1-3$, respectively. The check marked second scale gives the highest confidence value for the central pixel.

false alarm rate and considering larger neighborhoods. The generalized N.P. test for these steps is formulated as follows

$$
y_{m, l}^{\text {scal }} \underset{\mathbb{H}_{0}}{\stackrel{\mathbb{H}_{1}}{\gtrless}} \frac{\log \left(\gamma^{\text {scale }}\right)+N_{\text {scale }} X_{s}}{\log \left(\frac{X_{e}+X_{s}}{X_{e}}\right)},
$$

where $y_{m, l}^{\text {scale }}$ is the summation of the pixel values in the $N_{\text {scale }}=N(2 \times \text { scale }-1)^{2}$ pixels neighborhood around the pixel $[m, l]$ (note that other neighborhood expansion strategies with different shape and size can be also considered in this algorithm). Our confidence in the decision made on each scale is defined as the distance between the summation of measurements in the super-pixel and that of the threshold set by the GLRT:

$$
\text { Confidence }_{m, l}^{\text {scale }}=\left|y_{m, l}^{\text {scale }}-\frac{\log \left(\gamma^{\text {scale }}\right)+N_{\text {scale }} X_{s}}{\log \left(\frac{X_{e}+X_{s}}{X_{e}}\right)}\right| \text {. }
$$

Note that the optimal scale is not unique for all pixels, as finer scales are more suitable for pixels located on the texture or edge areas, and coarser scales are more suitable for the pixels located in flat areas. Therefore, we decide on the presence or absence of the object at a particular pixel based on the test result of the scale that shows the highest confidence value. The memory requirement of this technique is independent of the maximum scale number, since we only need to keep the original image, the last estimated image and the corresponding confidence values.

To have a better understanding of the proposed multi-scale GLRT technique and its performance, we set up an illustrative controlled imaging scenario. Fig.2(a) shows ideal (noiseless and deterministic) image of objects of different size and shape. To depict an experiment at the limit distance where the signal of interest is very weak, we consider an imaging scenario where the average number of received ballistic photons 
for each pixel is one photon. Fig.2(b) shows such Poissonian random signals (yet free of noise effects). Detection of such signals becomes more difficult when we consider the system noise as illustrated in Fig.2(c), where the Poisson noise variance is 40. Fig.2(d) is an image reconstructed by implementing the point-by-point single pixel detection techniques, considering a false alarm rate of 0.00125 , where none of the objects are correctly identified. On the other hand, Fig.2(e) is the result of exploiting the multi-scale GLRT technique, showing a considerably more accurate detection of such objects.

Fig.2(f) illustrates the scale from which each pixel in the final image of Fig.2(e) is selected. Note that as expected, the pixels in the flat area are selected from the coarser scales, while the pixels on the edge areas are selected from the finer scales. Fig.2(g) shows the confidence in the detection result (9) with respect to the corresponding pixels. This figure shows higher confidence levels in the flat, and less confidence in the edge areas. Also, in Fig.2(g) we see that the area with the lowest confidence is the place where most misclassifications happen. This is good news, since to increase the detection rate, we may opt to do a second (and very quick) round of scans, sampling only on these very low-confidence regions. In Fig.2(h), we plot the misclassification rates at each scale (blue line), and compare it to the overall multi-scale one (red line). These experimental plots show that the performance of the proposed pixelwise GLRT technique (depending on the noise level) is either very close or even better than the best fixed scale technique. In Fig.2(i), the performance of single pixel detection technique is compared with the multi-scale ones via their corresponding ROC curves (with 25 Monte Carlo experiments). Once again, the multi-scale technique shows the best or close to the best performance.

\section{CONCLUSION}

In this paper, we studied a technique for improving the quality of the ballistic images captured through turbid media.The novelty of this paper is in combining the recent advances in optical science with the novel image processing and statistical signal processing techniques.

\section{REFERENCES}

[1] K.M. Yoo and R.R. Alfano, “Time-resolved coherent and incoherent components of forward light scattering in random media," Optics Letters, vol. 15, no. 6, pp. 320-322, Mar. 1990.

[2] M. E. Zevallos, S. K. Gayen, M. Alrubaiee, and R. R. Alfano, "Time-gated backscattered ballistic light imaging of objects in turbid water," Applied Physics Letters, vol. 86, pp. 011115-1-011115-3, Jan. 2005.

[3] D. Contini, F. Martelli, and G. Zaccanti, "Photon migration through a turbid slab described by a model based on

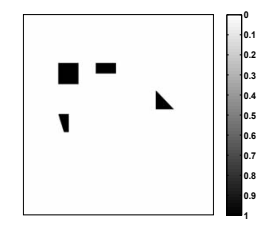

a

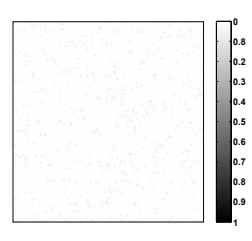

d

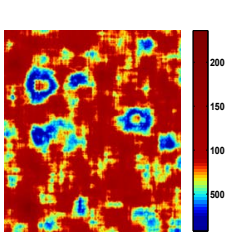

g

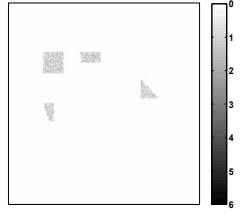

b

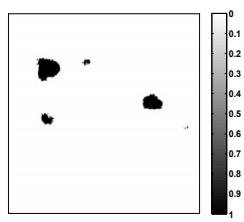

e

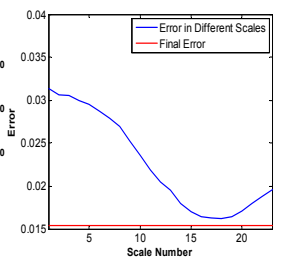

$\mathrm{h}$

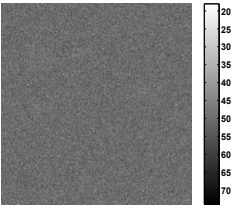

$\mathrm{c}$

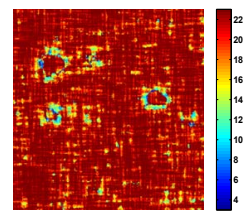

f

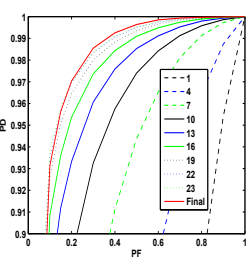

Fig. 2. An ideal deterministic and noise free image of four objects of different size and shapes is shown in (a). (b) shows the corresponding image as a Poissonian noise free stochastic signal, with $X_{s}=1$. (c) is the result of adding Poisson noise ( $X_{e}=40$ ) to (a). (d) is the result of the single pixel detection, and (e) is the result of the proposed multi-scale detection technique. (f) shows an image that corresponds to the selected scales for the image shown in (e), and (g) shows the corresponding confidence values. (h) shows the misclassification probability in different scales. ROC plots for the proposed multi-scale detection scenario are shown in (i). The numerical labels " $1,4, \ldots, 23$ ", correspond to the scale at which detection tests are performed, and the plot labeled "Final" represents the performance of the proposed multiscale (fused) technique.

diffusion approximation. i. theory," Applied Optics, vol. 36, no. 19, pp. 4587-4599, July 1997.

[4] W. Cai, S. K. Gayen, M. Xu, M. Zevallos, M. Alrubaiee, M. Lax, and R. R. Alfano, "Optical tomographic image reconstruction from ultrafast time-sliced transmission measurements," Appl. Opt., vol. 38, no. 19, pp. 42374246, July 1999.

[5] A.P. Gibson, J.C. Hebden, and S.R. Arridge, "Recent advances in diffuse optical imaging," Physics in Medicine And Biology, vol. 50, pp. R1-R43, Feb. 2005.

[6] S. M. Kay, Fundamentals of statistical signal processing:detection theory, vol. II, Prentice-Hall, Englewood Cliffs, New Jersey, 1998. 